Formal to Practical Security - LNCS 5458, pages 138-157. V. Cortier, C. Kirchner, M. Okada, and H. Sakurada Eds. Springer-Verlag.

\title{
New Anonymity Notions for Identity-Based Encryption
}

\author{
Malika Izabachène and David Pointcheval \\ Ecole Normale Supérieure - LIENS/CNRS/INRIA, France \\ Malika. Izabachene, David. Pointcheval\}@ens.fr
}

\begin{abstract}
Identity-based encryption is a very convenient tool to avoid key management. Recipient-privacy is also a major concern nowadays. To combine both, anonymous identity-based encryption has been proposed. This paper extends this notion to stronger adversaries (the authority itself). We discuss this new notion, together with a new kind of non-malleability with respect to the identity, for several existing schemes. Interestingly enough, such a new anonymity property has an independent application to password-authenticated key exchange. We thus come up with a new generic framework for password-authenticated key exchange, and a concrete construction based on pairings.
\end{abstract}

\section{Introduction}

Motivation. The idea of using identities instead of public keys in order to avoid the (costly) use of certificates comes from Shamir [19]. He indeed suggested Identity-based Encryption $(\mathcal{I B E})$, that would allow a user to encrypt a message using any string, that would specify the recipient, as encryption parameter, such that this recipient only can decrypt the ciphertext. Identity-based cryptography thus provides this interesting feature that one does not need authenticated public keys. Key managament is made simpler. Note however that a drawback is an authority that is required to generate the private keys for the users, according to their identities. This authority thus has the ability to decrypt any ciphertext. Privacy cannot be achieved with respect to this authority. Nevertheless, privacy of the plaintext is not the unique goal in cryptography, with encryption schemes. Privacy of the recipient may also be a requirement. Such a key-privacy notion has already been defined in the public-key setting in [3]. It has more recently been extended to the identity-based setting in [1], under the notion of anonymity. However, the security model in this $\mathcal{I B E}$ setting still trusts the authority. Whereas trusting the authority is intrinsic for privacy of the plaintext, it is not for the privacy of the recipient: a stronger anonymity notion is possible, with respect to the authority, but is it achievable for practical $\mathcal{I B E}$ ?

For efficiency reasons, the use of Key Encapsulation Mechanisms $\mathcal{K} \mathcal{E} \mathcal{M}$ have been shown as a preferable approach [21]. It consists in generating an ephemeral key and an encrypted version of the latter. The ephemeral key is thereafter used with a Data Encryption Method $\mathcal{D} \mathcal{E} \mathcal{M}$ to encrypt the message. In such a context, we are interested in the semantic security of the ephemeral key, and the anonymity of the recipient. In the identity-based context, Bentahar et al. [7] defined Identity-based Key Encapsulation Mechanisms $\mathcal{I} \mathcal{B}-\mathcal{K} \mathcal{E} \mathcal{M}$. An anonymity notion with respect to the authority would then be an interesting feature. Interestingly enough, this notion of anonymity with respect to the authority might have side applications. One of them is PAKE [6], for password-authenticated key exchange. Such a protocol allows two players to establish a private channel, using a short secret as a sole authentication means. The latter is thus subject to exhaustive search, but such a short secret is very convenient for human beings.

Related Work. The idea of identity-based encryption is due to Shamir [19], in 1984. The first goal was to simplify public key management. However, the first practical solutions appeared in 2001 only [10, 15]. Thereafter, many schemes have been proposed, based on pairing, factoring and lattices. Since such schemes were dealing with encryption, the main security notion was the semantic security [17]. 
Even if recipient-anonymity had already been addressed for public-key encryption [3] in 2001, anonymity for $\mathcal{I B E}$ has been proposed recently by Abdalla et al. [1], but as a simple extension of the previous public-key setting definition. In 2006, Gentry [16] and Boyen and Waters [12] presented the first anonymous $\mathcal{I B E}$ schemes without random oracles.

OUR CONTRIBUtions. As already noticed in [1], anonymity might have some side applications to searchable encryption. In this paper, we deal with anonymity for $\mathcal{I B}-\mathcal{K} \mathcal{E} \mathcal{M}$, even with respect to the authority, the so-called Key Anonymity with respect to the Authority and denoted KwrtA-Anonymity: we first provide a formal security model, and then we discuss this security notion with existing schemes. We also consider a new non-malleability notion for the identity, that we call identity-based non-malleability: if one encrypts a message (or a key) for user $U$, one has no idea about the value obtained by another user $U^{\prime}$, whatever the relation between $U$ and $U^{\prime}$ (or the identities) is.

Thereafter, we show that these security notions can also have side applications to password-authenticated key exchange. Such a KwrtA-anonymous and identity-based non-malleability $\mathcal{I B}$ - $\mathcal{K} \mathcal{E} \mathcal{M}$ scheme can indeed be plugged into a password-authenticated two-party key exchange protocol, in the same vein as the IPAKE construction [14] did with trapdoor hard-to-invert group isomorphisms. Our security result holds in a stronger security model than usual (with an adaptive selection of passive and active attacks, as in [18]), but the construction still assumes the random-oracle model [5], as in [14].

Eventually, we provide an $\mathcal{I} \mathcal{B}-\mathcal{K} \mathcal{E} \mathcal{M}$, that is both $K w r t A$-anonymous and identity-based non-malleable, in addition to the full-identity semantic security, against chosen-plaintext adversaries. This thus leads to a new password-authenticated two-party key exchange protocol.

\section{Anonymous Identity-Based Encryption}

Anonymity for public-key encryption schemes has first been introduced by Bellare et al. [3], under the key privacy security notion, and has been extended to identity-based encryption by Abdalla et al. [1].

In these papers, anonymity meant that even if the adversary chooses a message and two identities (or two public keys), and the challenger encrypts the message with one of the identities (or keys), the adversary cannot guess which one has actually been involved in the computation. This notion is quite strong for public-key encryption, but not that strong in the identity-based setting since it does not capture anonymity with respect to the authority that knows the master secret key, and even chooses the public parameters PK.

Unfortunately, the previous definitions cannot be trivially extended: the adversary can easily break anonymity if he knows the expected plaintext, and just hesitates between two identities, since he can decrypt any ciphertext. Anonymity can only be expected against the server if the plaintexts follow a nontrivial distribution. Since we will deal with key-encapsulation mechanisms, this non-trivial distribution is already implicit for the ephemeral keys.

This enhanced security notion will be called Key Anonymity with respect to the Authority and denoted KwrtA-Anonymity. This section defines precisely this notion for identity-based key encapsulation mechanisms.

\subsection{Identity-Based Encryption and Key Encapsulation Mechanisms}

We first review the definitions of identity-based encryption, and more specifically of identity-based key encapsulation mechanisms [7]. In the following, we assume that identities are bit strings in a dictionary Dic. 
Definition 1 (Identity-Based Encryption). An $\mathcal{I B E}$ scheme is specified by four algorithms:

Setup $_{\mathrm{IBE}}\left(1^{\lambda}\right)$. Takes as input a security parameter $\lambda$. It outputs the public parameters PK, as well as a master secret key MK.

$\operatorname{Extract}_{\mathrm{IBE}}(\mathrm{MK}, \mathrm{ID})$. Takes as input the master secret key MK, and the identity ID of the user. It outputs the user's decryption key usk.

Encrypt $_{\mathrm{IBE}}(\mathrm{PK}, \mathrm{ID}, M)$. Takes as input the public parameter $\mathrm{PK}$, the identity of the recipient, and a message $M$ to be encrypted. It outputs a ciphertext.

$\operatorname{Decrypt}_{\mathrm{IBE}}($ usk, $c$ ). Takes as input the user's decryption key and a ciphertext $c$. It outputs the decryption or $\perp$, if the ciphertext is not valid.

In [20] Shoup proposed a more efficient framework for public-key encryption, the so-called KEM/DEM, for key encapsulation mechanism/data encapsulation method. More recently, Bentahar et al. [7] extended this concept to the identity-based setting, and therefore proposed some constructions of $\mathcal{I} \mathcal{B}-\mathcal{K} \mathcal{E} \mathcal{M}$ semantically secure. We will use the following formalism:

Definition 2 (Identity-Based Key Encapsulation Mechanism).

An $\mathcal{I B}-\mathcal{K} \mathcal{E} \mathcal{M}$ scheme is specified by the following four algorithms:

$\operatorname{Setup}_{\mathrm{IBK}}\left(1^{\lambda}\right)$. Takes as input a security parameter $\lambda$. It outputs the public parameters PK, as well as a master secret key MK.

Extract $_{\mathrm{IBK}}(\mathrm{MK}, \mathrm{ID})$. Takes as input the master secret key MK and an identity ID of the user. It outputs the user's decryption key usk.

Encaps $_{\mathrm{IBK}}(\mathrm{PK}, \mathrm{ID})$. Takes as input the public parameters PK and the identity of the recipient. It outputs a pair $(K, c)$, where $K$ is the ephemeral session key and $c$ is the encapsulation of that key.

$\operatorname{Decaps}_{\mathrm{IBK}}($ usk,, ). Takes as input the user's decryption key usk and a ciphertext $c$. It outputs the key $K$ encapsulated in $c$ or $\perp$, if the ciphertext is not valid.

We also formally define the function $\operatorname{Decaps}_{\mathrm{IBK}}(\mathrm{ID}, c)$, which takes as input a user identity ID and a ciphertext $c$. It first extracts the decryption key usk associated to ID, and then decapsulates $c$ under usk.

We first review the notion of semantic security for $\mathcal{I B}-\mathcal{K} \mathcal{E} \mathcal{M}$, then we deal with anonymity, and an additional security notion, that we call identity-based non-malleability.

\subsection{Security Notions}

We directly describe the security notions for identity-based key encapsulation mechanisms, but one can easily derive them for identity-based encryption.

Semantic Security. The semantic security formalizes the privacy of the key. The security game, in the strongest security model (i.e. chosen-ciphertext and full-identity attacks) is the following one:

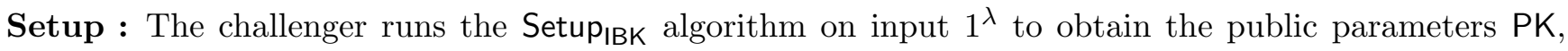
and the master secret key MK. It publishes PK.

Find stage: The adversary $\mathcal{A}$ adaptively issues the following queries:

- Extract query on input an ID: The challenger runs the Extract algorithm on input (MK, ID), and provides the associated decryption key usk.

- Decaps query on input an ID and a ciphertext $c$ : The challenger first extracts the decryption key for ID, and then decrypts the ciphertext $c$ with this key. It outputs the resulting ephemeral key, or $\perp$. 
$\mathcal{A}$ outputs a target identity ID*, on which no Extract-query has been asked.

Challenge: The challenger randomly gets $\left(K_{0}, c^{*}\right) \leftarrow \operatorname{Encaps}_{\mathrm{IBK}}\left(\mathrm{PK}, \mathrm{ID}^{*}\right)$ and $\left(K_{1}, c^{\prime}\right) \leftarrow \operatorname{Encaps}_{\mathrm{IBK}}\left(\mathrm{PK}, \mathrm{ID}^{*}\right)$. It flips a bit $b$ and outputs $\left(K_{b}, c^{*}\right)$.

Guess stage: The adversary can issue the same queries as in the Find stage, with the restriction that no Extract-query on input ID* and no Decaps-query on input $\left(\mathrm{ID}^{*}, c^{*}\right)$ can be asked. The adversary finally outputs its guess $b^{\prime} \in\{0,1\}$ for $b$.

We then define the advantage of $\mathcal{A}$ in breaking the Semantic Security of an $\mathcal{I B}-\mathcal{K} \mathcal{E} \mathcal{M}$ scheme with its ability in deciding whether it actually received the real ephemeral key associated to $c^{*}$ or a random one. We denote this security notion by IND, which can thereafter be combined with various oracle accesses, in order to define selective/full-identity and chosen plaintext/ciphertext attacks. More formally, we want the advantage below, to be negligible:

$$
\operatorname{Adv}_{\mathrm{IBK}}^{\text {ind }}(\mathcal{A})=2 \times \operatorname{Pr}_{b}\left[\begin{array}{c}
(\mathrm{PK}, \mathrm{MK}) \leftarrow \operatorname{Setup}_{\mathrm{IBK}}\left(1^{\lambda}\right) ;\left(\mathrm{ID}^{*}, s\right) \leftarrow \mathcal{A}_{1}(\mathrm{PK}) \\
\left(K_{0}, c^{*}\right) \leftarrow \operatorname{Encaps}_{\mathrm{IBK}}\left(\mathrm{PK}, \mathrm{ID}^{*}\right) ; \\
\left(K_{1}, c^{\prime} \leftarrow \operatorname{Encaps}_{\mathrm{IBK}}\left(\mathrm{PK}, \mathrm{ID}^{*}\right)\right. \\
b^{\prime} \leftarrow \mathcal{A}_{2}\left(K_{b}, c^{*}, s\right): b=b^{\prime}
\end{array}\right]-1 .
$$

In the following, we will need a very weak notion, that we call weak semantic security, during which attack that adversary has to choose in advance the target identity ID* (selective-ID), and has no oracle access at all: no Decaps queries, and no Extract queries.

ANONYMITY. Anonymity against $\mathcal{I B E}$ means that for a chosen plaintext, and given a ciphertext $c$ encrypted under $\mathrm{ID}_{0}$ or $\mathrm{ID}_{1}$ of adversary's choice, the adversary should not be able to decide which identity has been involved. With an appropriate $\mathcal{D E} \mathcal{M}$ encryption scheme, the key encapsulation anonymity version can be defined as follows:

Setup: The challenger runs Setup $\mathrm{IBK}_{\mathrm{BK}}$ on input $1^{\lambda}$ to obtain the public parameters PK, and the master secret key MK. It publishes PK.

Find stage: The adversary $\mathcal{A}$ adaptively issues Extract and Decaps queries. $\mathcal{A}$ outputs two identities $\mathrm{ID}_{0}, \mathrm{ID}_{1}$, on which no Extract-query has been asked before.

Challenge: The challenger randomly selects $b \in\{0,1\}$ and gets an encapsulated pair $\left(K^{*}, c^{*}\right)$ under $\mathrm{ID}_{b}$. It returns $\left(K^{*}, c^{*}\right)$.

Guess stage: The adversary can issue the same queries as in the Find stage, subject to the restriction that no Extract-query is allowed to be asked on $\mathrm{ID}_{0}$ or $\mathrm{ID}_{1}$, and no Decaps-query can be asked on input $\left(\mathrm{ID}_{0}, c^{*}\right)$, or $\left(\mathrm{ID}_{1}, c^{*}\right)$. It finally outputs its guess $b^{\prime} \in\{0,1\}$ for $b$.

We say that an $\mathcal{I B}-\mathcal{K} \mathcal{E} \mathcal{M}$ scheme provides key-anonymity if the advantage of $\mathcal{A}$ in deciding which identity is actually involved in the above experiment is negligible:

$$
\operatorname{Adv}_{\mathrm{IBK}}^{\text {anon }}(\mathcal{A})=2 \times \operatorname{Pr}_{b}\left[\begin{array}{c}
(\mathrm{PK}, \mathrm{MK}) \leftarrow \operatorname{Setup}_{\mathrm{IBK}}\left(1^{\lambda}\right) ; \\
\left(\mathrm{ID}_{0}, \mathrm{ID}_{1}, s\right) \leftarrow \mathcal{A}_{1}(\mathrm{PK}) \\
\left(K^{*}, c^{*}\right) \leftarrow \operatorname{Encaps}_{\mathrm{IBK}}\left(\mathrm{PK}, \mathrm{ID}_{b}\right) ; \\
b^{\prime} \leftarrow \mathcal{A}_{2}\left(K^{*}, c^{*}, s\right): b=b^{\prime}
\end{array}\right]-1
$$

As already noticed, this anonymity notion does not provide any security with respect to the authority, since the above security notion assumes that the adversary has no idea about MK.

KwrtA-Anonymity. We therefore enhance the previous security model, in order to consider the authority as a possible adversary. However, it is clear that given $\left(K^{*}, c^{*}\right)$, the authority can check the involved ID. We thus truncate the input to $c^{*}$ only: 
Find stage: The adversary generates (valid, see below) public parameters PK. $\mathcal{A}$ outputs PK and two identities $I D_{0}, I D_{1}$.

Challenge : The challenger randomly selects $b \in\{0,1\}$, and generates a ciphertext for $\operatorname{ID}_{b},\left(K^{*}, c^{*}\right) \leftarrow$ Encaps $_{\mathrm{IBK}}\left(\mathrm{PK}, \mathrm{ID}_{b}\right)$. It outputs $c^{*}$.

Guess stage: The adversary finally outputs its guess $b^{\prime} \in\{0,1\}$.

We say that an $\mathcal{I B}-\mathcal{K} \mathcal{E} \mathcal{M}$ scheme provides Key Anonymity with respect to the Authority (denoted KwrtAAnonymity) if the advantage of $\mathcal{A}$ in deciding which identity is involved in the experiment above is negligible:

$$
\operatorname{Adv}_{\mathrm{IBK}}^{\mathrm{kwrta}-\operatorname{anon}}(\mathcal{A})=2 \times \operatorname{Pr}_{b}\left[\begin{array}{c}
\left(\mathrm{PK}, \mathrm{ID}_{0}, \mathrm{ID}_{1}, s\right) \leftarrow \mathcal{A}_{1}\left(1^{\lambda}\right) \text { s.t. Valid } \mathrm{VBK}_{\mathrm{BK}}(\mathrm{PK}) \\
\left(K^{*}, c^{*}\right) \leftarrow \operatorname{Encaps}_{\mathrm{IBK}}\left(\mathrm{PK}, \mathrm{ID}_{b}\right) ; \\
b^{\prime} \leftarrow \mathcal{A}_{2}\left(c^{*}, s\right): b=b^{\prime}
\end{array}\right]-1
$$

We emphasis that in the above experiment, the adversary has to generate valid public parameters PK. Note that against KwrtA-Anonymity (vs. anonymity), on the one hand, the new adversary may know the master key MK, but on the other hand, it must make its decision from $c^{*}$ only. Therefore, these two security notions are not really comparable. Furthermore, since the adversary generates PK, one has to be able to check the honest generation. In some cases, PK is a truly random value, without redundancy; in some other cases, appropriate redundancy should be proven. We thus define an additional algorithm:

Valid $_{\mathrm{IBK}}(\mathrm{PK})$. Takes as input the public parameters PK, and checks whether they satisfy the required properties.

IDENTITY-BASED Non-MALleABILITy. In the application we will study later, a new security notion for identity-based encryption will appear. It basically states that when one sends a ciphertext to a user ID, one has no idea how user ID' will decrypt it, even for identities chosen by the adversary. This means that when one computes an encapsulation, it provides an ephemeral session key with a unique recipient, and not several secret keys with several partners. We define the identity-based non-malleability game as follows:

Setup: The challenger runs Setup $\mathrm{IBK}_{\mathrm{BK}}$ on input $1^{\lambda}$ to obtain the public parameters $\mathrm{PK}$, and the master secret key MK. It publishes PK.

Attack: The adversary $\mathcal{A}$ adaptively issues Extract and Decaps queries, and outputs a ciphertext $c$, and two pairs $\left(K_{0}, \mathrm{ID}_{0}\right)$, and $\left(K_{1}, \mathrm{ID}_{1}\right)$.

The adversary wins this game if the two formal equalities hold:

$$
K_{0}=\operatorname{Decaps}_{\mathrm{IBK}}\left(\mathrm{ID}_{0}, c\right) \text { and } K_{1}=\operatorname{Decaps}_{\mathrm{IBK}}\left(\mathrm{ID}_{1}, c\right) .
$$

We thus define the success of $\mathcal{A}$ in breaking the Identity-based Non-Malleability of an $\mathcal{I} \mathcal{B}-\mathcal{K} \mathcal{E} \mathcal{M}$ scheme by:

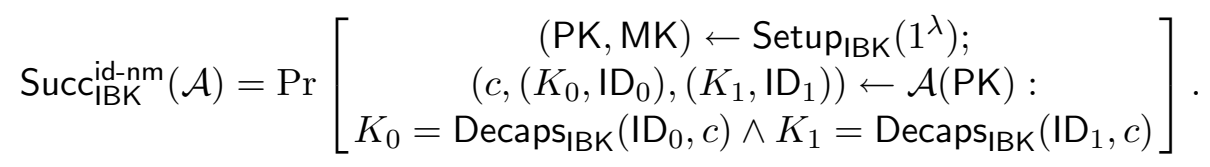

Note that this security notion is for a normal user, and not for the authority itself. Indeed, it would clearly be incompatible with KwrtA-Anonymity. 


\section{Anonymous and Non-Malleable $\mathcal{I B}-\mathcal{K} \mathcal{E} \mathcal{M}$}

Since the first practical $\mathcal{I B E}$ schemes, new features, and new efficient/security criteria have been defined. An efficient anonymous $\mathcal{I B E}$ with a tight security proof in the standard model is one of the open problems. In this section, we first review some candidates, and then propose a new scheme that satisfies all the above requirements: semantic security, various anonymity notions and identity-based non-malleability.

\subsection{Backgrounds on Pairings}

Let $\mathbb{G}_{1}$ and $\mathbb{G}_{2}$ be two cyclic groups of large prime order $p$. We suppose that these two groups are equipped with a pairing, i.e. a non-degenerated and efficiently computable bilinear map $\hat{e}: \mathbb{G}_{1} \times \mathbb{G}_{2} \rightarrow \mathbb{G}_{T}$. In the following, we use multiplicative notation for $\mathbb{G}_{1}$ and $\mathbb{G}_{2}: \hat{e}\left(g_{1}^{a}, g_{2}^{b}\right)=\hat{e}\left(g_{1}, g_{2}\right)^{a b}$, for all $a, b \in \mathbb{Z}_{p}$, and any $g_{1} \in \mathbb{G}_{1}$ and $g \in \mathbb{G}_{2}$. For the sake of generality, we consider the asymmetric case, where $\mathbb{G}_{1} \neq \mathbb{G}_{2}$, but most of the schemes below also apply in the symmetric setting, where $\mathbb{G}_{1}=\mathbb{G}_{2}$.

\subsection{Diffie-Hellman Assumptions}

The co-CDH-Problem. Let $g_{1}$ and $g_{2}$ two generators of $\mathbb{G}_{1}$ and $\mathbb{G}_{2}$ respectively. We define the co-DiffieHellman value co- $\mathrm{CDH}_{g_{1}, g_{2}}(u)$, for $u=g_{1}^{x} \in \mathbb{G}_{1}$, the element $v=g_{2}^{x} \in \mathbb{G}_{2}$.

The co- $\mathrm{CDH}_{\mathbb{G}_{1}, \mathbb{G}_{2}}$ problem can be formalized as follows: given $g_{1}, u \in \mathbb{G}_{1}$ and $g_{2} \in \mathbb{G}_{2}$, output $v=$ co- $\mathrm{CDH}_{g_{1}, g_{2}}(u)$. We define the success probability of $\mathcal{A}$ in breaking the co- $\mathrm{CDH}_{\mathbb{G}_{1}, \mathbb{G}_{2}}$-problem as:

$$
\operatorname{Succ}_{\mathbb{G}_{1}, \mathbb{G}_{2}}^{\mathrm{co}-\mathrm{cdh}}(\mathcal{A})=\operatorname{Pr}\left[g_{1} \stackrel{R}{\leftarrow} \mathbb{G}_{1} ; g_{2} \stackrel{R}{\leftarrow} \mathbb{G}_{2}, x \stackrel{R}{\leftarrow} \mathbb{Z}_{p} ; v \leftarrow \mathcal{A}\left(g_{1}, g_{2}, g_{1}^{x}\right): v=g_{2}^{x}\right] .
$$

Note that when $\mathbb{G}_{1}=\mathbb{G}_{2}=\mathbb{G}$, the co-CDH $\mathbb{G}_{\mathbb{G}, \mathbb{G}}$-problem is exactly the usual Computational Diffie-Hellman Problem in $\mathbb{G}$, which can still be difficult. However, the decisional version is easy, granted the pairing.

We can indeed define the co-DH $\mathbb{G}_{1}, \mathbb{G}_{2}$-language of the quadruples $(a, b, c, d) \in \mathbb{G}_{1} \times \mathbb{G}_{2} \times \mathbb{G}_{1} \times \mathbb{G}_{2}$, such that $d=\mathrm{co}-\mathrm{CDH}_{a, b}(c)$.

The Common co-CDH-Problem. Given two elements, it is simple to complete a co-CDH-quadruple $\left(g_{1}, g_{2}, u, v\right)$. However, finding two such quadruples with constraints may not be simple. We thus define a new problem, called the Common co-CDH-Problem, as follows: Given $g, h \in \mathbb{G}$, and $V \in \mathbb{G}_{T}$, output $k_{0} \neq k_{1} \in \mathbb{Z}_{p}, K_{0}, K_{1} \in \mathbb{G}_{T}$ and a common $c \in \mathbb{G}$, such that:

$$
\left(g h^{k_{0}}, V, c, K_{0}\right),\left(g h^{k_{1}}, V, c, K_{1}\right) \in \mathrm{co}^{-\mathrm{DH}_{\mathbb{G}, \mathbb{G}_{T}}} .
$$

We define the success of $\mathcal{A}$ in breaking the Common-co-CDH $\mathbb{G}_{\mathbb{e}, \hat{e}}-$ Problem as:

$$
\operatorname{Succ}_{\mathbb{G}, \hat{e}}^{\text {common-co-cdh }}(\mathcal{A})=\operatorname{Pr}\left[\begin{array}{c}
g, h \in \mathbb{G} ; V \in \mathbb{G}_{T} ;\left(c, k_{0}, k_{1}, K_{0}, K_{1}\right) \leftarrow \mathcal{A}(g, h, V): \\
k_{0} \neq k_{1} \wedge\left(g h^{k_{0}}, V, c, K_{0}\right) \in \operatorname{co-DH} H_{\mathbb{G}, \mathbb{G}_{T}} \\
\wedge\left(g h^{k_{1}}, V, c, K_{1}\right) \in \operatorname{co-DH}_{\mathbb{G}, \mathbb{G}_{T}}
\end{array}\right]
$$

The CBDH-Problem. Diffie-Hellman variants have been proposed in groups equipped with pairings, and namely in the symmetric case: let $g$ be a generator of $\mathbb{G}$. We define the Bilinear Diffie-Hellman value of $g^{x}, g^{y}, g^{z}$, for $x, y, z \in \mathbb{Z}_{p}$, in base $g$, the element $V=\hat{e}(g, g)^{x y z} \in \mathbb{G}_{T}$.

The $\mathrm{CBDH}_{\mathbb{G}, \hat{e}}$ problem can be formalized as follows: given $g, X=g^{x}, Y=g^{y}, Z=g^{z} \in \mathbb{G}$, output $V=\hat{e}(g, g)^{x y z}$. We define the success probability of $\mathcal{A}$ in breaking the $\mathrm{CBDH}_{\mathbb{G}, \hat{e}}$-problem as:

$$
\operatorname{Succ}_{\mathbb{G}, \hat{e}}^{\mathrm{cbdh}}(\mathcal{A})=\operatorname{Pr}\left[g \stackrel{R}{\leftarrow} \mathbb{G} ; x, y, z \stackrel{R}{\leftarrow} \mathbb{Z}_{p} ; V \leftarrow \mathcal{A}\left(g, g^{x}, g^{y}, g^{z}\right): v=\hat{e}(g, g)^{x y z}\right] .
$$


The DBDH-Problem. The decisional version can then be intractable too: given $g, X=g^{x}, Y=g^{y}, Z=$ $g^{z} \in \mathbb{G}$, and $V \in \mathbb{G}_{T}$, decide whether $V=\hat{e}(g, g)^{x y z}$, or not. We define the advantage of $\mathcal{A}$ in breaking the $\mathrm{DBDH}_{\mathbb{G}, \hat{e}}-$ problem as:

$$
\begin{aligned}
\operatorname{Adv}_{\mathbb{G}, \hat{e}}^{\mathrm{dbdh}}(\mathcal{A}) & =\operatorname{Pr}\left[g \stackrel{R}{\leftarrow} \mathbb{G} ; x, y, z \stackrel{R}{\leftarrow} \mathbb{Z}_{p} ; V=\hat{e}(g, g)^{x y z}: 1 \leftarrow \mathcal{A}\left(g, g^{x}, g^{y}, g^{z}, V\right)\right] \\
& -\operatorname{Pr}\left[g \stackrel{R}{\leftarrow} \mathbb{G} ; x, y, z \stackrel{R}{\leftarrow} \mathbb{Z}_{p} ; V \stackrel{R}{\leftarrow} \mathbb{G}_{T}: 1 \leftarrow \mathcal{A}\left(g, g^{x}, g^{y}, g^{z}, V\right)\right] .
\end{aligned}
$$

The Successive-Power Version. For our scheme to be semantically secure, we will need a stronger variant of the above DBDH problem, given access to a sequence of powers, similarly to the Strong DiffieHellman problem [9]: More precisely, given $g, g^{x}, g^{y}, g^{z}$, and $g^{z / x}, g^{z / x^{2}}, \ldots, g^{z / x^{q}}$, as well as $V$, from some $V \in \mathbb{G}_{T}$, where $q$ is a parameter, decide whether $V=\hat{e}(g, g)^{x y z}$, or a random element. We define the

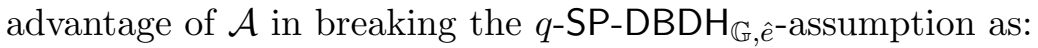

$$
\begin{aligned}
\operatorname{Adv}_{\mathbb{G}, \hat{e}}^{q-\operatorname{spdbdh}}(\mathcal{A})=\operatorname{Pr}\left[\begin{array}{c}
g \stackrel{R}{\leftarrow} \mathbb{G} ; x, y, z \stackrel{R}{\leftarrow} \mathbb{Z}_{p} ; V=\hat{e}(g, g)^{x y z}: \\
1 \leftarrow \mathcal{A}\left(g, g^{x}, g^{y}, g^{z}, g^{z / x}, \cdots, g^{z / x^{q}}, V\right)
\end{array}\right] \\
-\operatorname{Pr}\left[\begin{array}{c}
g \stackrel{R}{\leftarrow} \mathbb{G} ; x, y, z \stackrel{R}{\leftarrow} \mathbb{Z}_{p} ; V \stackrel{R}{\leftarrow} \mathbb{G}_{T}: \\
1 \leftarrow \mathcal{A}\left(g, g^{x}, g^{y}, g^{z}, g^{z / x}, \cdots, g^{z / x^{q}}, V\right)
\end{array}\right] .
\end{aligned}
$$

It is clear that such a sequence of powers should not provide much information to the adversary. And thus, for any polynomial-time adversary $\mathcal{A}$, the above advantage is negligible. We provide the proofs that our two new problems are intractable for generic adversaries in the Appendix A.

\subsection{Previous $\mathcal{I}$ BE Schemes}

Let us review several $\mathcal{I B E}$, and see which properties they satisfy. For the sake of simplicity, for all of them, we review the key encapsulation mechanisms. In several schemes, we will need a deterministic map $F$ from identities onto the group $\mathbb{G}$, possibly with parameter PK.

The Boneh-Franklin Scheme [10]. In this scheme, MK $=s \stackrel{R}{\leftarrow} \mathbb{Z}_{p}$ and $\mathrm{PK}=g^{s}$. The map $F($ ID) is independent of PK. This is a function onto $\mathbb{G}$, modeled as a random oracle in the security analysis. The ciphertext $c=g^{r} \in \mathbb{G}$ corresponds to the key $K=\hat{e}(F(\mathrm{ID}), \mathrm{PK})^{r}=\mathrm{BDH}_{g}(\mathrm{PK}, c, F(\mathrm{ID}))=\hat{e}\left(\right.$ usk $\left._{\mathrm{ID}}, c\right)$, where usk ID $=F(\text { ID })^{s}=$ co- $\mathrm{CDH}_{g, F(\mathrm{ID})}(\mathrm{PK}) \in \mathbb{G}$.

It is quite similar to the ElGamal encryption, and thus the semantic security relies on the DBDH $_{\mathbb{G}, \hat{e}}$, but against chosen-plaintext attacks only, in the random oracle model, even with access to the Extractquery, which is similar to the Boneh-Lynn-Shacham signature [11] (secure against chosen-message attacks under the $\mathrm{CDH}_{\mathbb{G}}$ problem).

Since the ciphertext is totally independent of the identity, this scheme is KwrtA-anonymous, in the information-theoretical sense. Nevertheless, the basic anonymity is similar to the semantic security, and relies on the $\mathrm{DBDH}_{\mathbb{G}, \hat{e}}$. However, since the ciphertext does not involve the identity, it is easy to break the identity-based non-malleability: knowing $r$ and $c=g^{r}$, one easily computes $K=\mathrm{BDH}_{g}(\mathrm{PK}, c, F(\mathrm{ID}))=$ $\hat{e}(F(\mathrm{ID}), \mathrm{PK})^{r}$, for any ID of ones choice.

The Boneh-Boyen Scheme [8]. In this scheme, $\alpha \stackrel{R}{\leftarrow} \mathbb{Z}_{p}, g, g_{2}, h \stackrel{R}{\leftarrow} \mathbb{G}$, and PK $=\left(g, g_{1}=g^{\alpha}, g_{2}, h\right)$, while $\mathrm{MK}=g_{2}^{\alpha}$. The map $F_{\mathrm{PK}}$ is defined by $F_{\mathrm{PK}}(\mathrm{ID})=g_{1}^{\mathrm{ID}} \cdot h$. The ciphertext $c=\left(g^{s}, F_{\mathrm{PK}}(\mathrm{ID})^{s}\right)$ corresponds to the key

$$
K=\hat{e}\left(g_{1}, g_{2}\right)^{s}=\hat{e}\left(c_{1}, \text { usk }_{2}\right) / \hat{e}\left(\text { usk }_{1}, c_{2}\right),
$$


if one gets usk $\mathrm{ID}_{\mathrm{D}}=\left(g^{r}, \mathrm{MK} \cdot F_{\mathrm{PK}}(\mathrm{ID})^{r}\right)$, for any $r \stackrel{R}{\leftarrow} \mathbb{Z}_{p}$.

As above, the semantic security relies on the $\mathrm{DBDH}_{\mathbb{G}, e,}$ assumption, in the standard model, but against selective-ID chosen-plaintext attacks, even with access to the Extract-query (the underlying signature scheme is selective-forgery secure against chosen-message attacks under the CBDH assumption).

However, because of the redundancy in the ciphertext, which matches with one identity only, this scheme is not anonymous: one just has to check, for a candidate ID, and a ciphertext $c=\left(c_{1}, c_{2}\right)$, whether $\left(g, F_{\mathrm{PK}}(\mathrm{ID}), c_{1}, c_{2}\right)$ is a Diffie-Hellman tuple, by $\hat{e}\left(c_{1}, F_{\mathrm{PK}}(\mathrm{ID})\right) \stackrel{?}{=} \hat{e}\left(c_{2}, g\right)$. Since this attack did not need a candidate key $K$, a fortiori, this scheme is not KwrtA-anonymous.

On the other hand, since the ciphertext focuses to a specific recipient, one has no idea how another ID' would decrypt it, because of its randomness $r^{\prime}$ in the decryption key: for wrong user, with usk' $=$ $\left(g^{r^{\prime}}, g_{2}^{\alpha} F_{\mathrm{PK}}\left(\mathrm{ID}^{\prime}\right)^{r^{\prime}}\right)$, and $c=\left(g^{s}, F_{\mathrm{PK}}\left(\mathrm{ID}^{\prime}\right)^{s^{\prime}}\right)\left(s^{\prime} \neq s\right.$ since $\mathrm{ID}^{\prime}$ is not the intended recipient $), K^{\prime}=K \times$ $H^{r^{\prime}}$, for $H \neq 1$, and $r^{\prime}$ totally random. Therefore, it is identity-based non-malleable in the informationtheoretical sense.

The Gentry Scheme [16]. In 2006, two schemes have been proposed, with provable anonymity. Gentry's scheme is one of them: $g, h \stackrel{R}{\leftarrow} \mathbb{G}$ and $\alpha \stackrel{R}{\leftarrow} \mathbb{Z}_{p}$. The public parameters are PK $=\left(g, g_{1}=g^{\alpha}, h\right)$ and $\mathrm{MK}=\alpha$. The map $F_{\mathrm{PK}}$ is defined by $F_{\mathrm{PK}}(\mathrm{ID})=g_{1} \cdot g^{-\mathrm{ID}}=g^{\alpha-\mathrm{ID}}$. The ciphertext $c=\left(F_{\mathrm{PK}}(\mathrm{ID})^{s}, \hat{e}(g, g)^{s}\right)$ is the encapsulation of $K=\hat{e}(g, h)^{s}$, and thus, setting $\left(\right.$ usk $_{1}$, usk $\left._{2}\right)=\left(r,\left(h g^{-r}\right)^{1 /(\alpha-\mathrm{ID})}\right)$, for any $r \stackrel{R}{\leftarrow} \mathbb{Z}_{p}$, $K=\hat{e}\left(c_{1}\right.$, usk $\left._{2}\right) \cdot c_{2}{ }^{\text {usk }_{1}}$.

The scheme is semantically secure and anonymous against chosen plaintext attacks, even with access to the Extract-query, under the truncated decisional augmented bilinear Diffie-Hellman exponent assumption (see [16] for details).

However, the scheme is not KwrtA-anonymous, since using bilinear maps combined with the redundancy inside the ciphertext provides a test for any target identity ID', since knowing $\alpha$, $\mathcal{A}$ can test whether

$$
c_{2}{ }^{\alpha-\mathrm{ID}^{\prime}}=e(g, g)^{s\left(\alpha-\mathrm{ID}^{\prime}\right)} \stackrel{?}{=} e\left(c_{1}, g\right)=e\left(g^{s\left(\alpha-\mathrm{ID}^{\prime}\right)}, g\right) .
$$

Since the ciphertext is specific to the recipient, $\mathcal{A}$ has no idea how an other ID' $\operatorname{decrypts} c=\left(c_{1}=\right.$ $\left.F_{\mathrm{PK}}\left(\mathrm{ID}^{\prime}\right)^{s^{\prime}}, c_{2}=e(g, g)^{s}\right)$, since

$$
K^{\prime}=\hat{e}\left(c_{1}, \text { usk }_{2}^{\prime}\right) \cdot c_{2}{ }^{\text {skk }_{1}^{\prime}}=K \cdot\left(\hat{e}(g, g)^{\text {usk }_{1}^{\prime}} / \hat{e}(g, h)\right)^{s-s^{\prime}},
$$

is a random element in $\mathbb{G}_{T}$. Thus, the scheme is identity-based non-malleable in the information-theoretical sense.

The Boyen-Whters scheme [13]. Boyen and Waters proposed another provably anonymous scheme: $\omega, t_{1}, t_{2}, t_{3}$ and $t_{4} \stackrel{R}{\leftarrow} \mathbb{Z}_{p}$ are set to be the master secret key and $\Omega=\hat{e}(g, g)^{t_{1} \cdot t_{2} \cdot \omega}, g, g_{0}, g_{1}, v_{1}=g^{t_{1}}, v_{2}=$ $g^{t_{2}}, v_{3}=g^{t_{3}}$ are the public parameters PK, with $g$ a random generator of $\mathbb{G}$ and $g_{0}, g_{1} \stackrel{R}{\leftarrow} \mathbb{G}$. The map $F_{\mathrm{PK}}$ is defined by $F_{\mathrm{PK}}(\mathrm{ID})=g_{0} \cdot$ ID. To encrypt a key, one chooses a random $s \in \mathbb{Z}_{p}$ and sets $K=\Omega^{s}$, its encapsulation has the following form: $c=\left(c_{0}, c_{1}, c_{2}, c_{3}, c_{4}\right)$, with $c_{0}=F_{\mathrm{PK}}(\mathrm{ID})^{s}, c_{1}=v_{1}^{s-s_{1}}, c_{2}=v_{2}^{s_{1}}$, $c_{3}=v_{3}^{s-s_{2}}$, and $c_{4}=v_{4}^{s_{2}}$. To decapsulate the key, one has to compute

$$
\begin{aligned}
K^{-1} & =\Omega^{-s}=\hat{e}(g, g)^{-\omega t_{1} t_{2} s} \\
& =\hat{e}\left(c_{0}, \text { usk }_{0}\right) \times \hat{e}\left(c_{1}, \text { usk }_{1}\right) \times \hat{e}\left(c_{2}, \text { usk }_{2}\right) \times \hat{e}\left(c_{3}, \text { usk }_{3}\right) \times \hat{e}\left(c_{4}, \text { usk }_{4}\right)
\end{aligned}
$$

with usk $_{\mathrm{ID}}=\left(\right.$ usk $_{0}$, usk $_{1}$, usk $_{2}$, usk $_{3}$, usk $\left._{4}\right)$, where:

$$
\begin{array}{ll}
\text { usk }_{0}=g^{r_{1} t_{1} t_{2}+r_{2} t_{3} t_{4}} & \\
\text { usk }_{1}=g^{-\omega t_{2}} F_{\mathrm{PK}}(\mathrm{ID})^{-r_{1} t_{2}} & \text { usk }_{2}=g^{-\omega t_{1}} F_{\mathrm{PK}}(\mathrm{ID})^{-r_{1} t_{1}} \\
\text { usk }_{3}=F_{\mathrm{PK}}(\mathrm{ID})^{-r_{2} t_{4}} & \text { usk }_{4}=F_{\mathrm{PK}}(\mathrm{ID})^{-r_{2} t_{3}}
\end{array}
$$


for any $r_{1}, r_{2} \stackrel{R}{\leftarrow} \mathbb{Z}_{p}$. This scheme is semantically secure under $\mathrm{DBDH}_{\mathbb{G}, \hat{e}}$, and anonymous under the decision linear assumption (we do not give more details since this scheme is totally different from ours below. The reader is refereed to [13]). However, it is not KwrtA-anonymous: since knowing the master key and given a ciphertext $c=\left(c_{0}, c_{1}, c_{2}, c_{3}, c_{4}\right)$, one can decide for a target identity whether $c_{0}, c_{1}, c_{2}$ or/and $c_{0}, c_{3}, c_{4}$ is a linear tuple in basis $v_{0}, v_{1}, v_{2}$ and $v_{0}, v_{3}, v_{4}$ respectively.

Since the key is completely independent of the identity and $c_{0}$ is determined by the identity (among other elements), the same argument than for the two previous schemes holds: it is identity-based nonmalleable in an information-theoretically sense.

Note that for all the above schemes, the public parameters consist of independent elements in appropriate groups. The validity check Valid $\mathrm{IBK}_{\mathrm{BK}}(\mathrm{PK})$ is thus trivial.

\subsection{Our Scheme}

None of the previous schemes satisfies both KwrtA-anonymity and identity-based non-malleability. In this section, we describe our scheme, and show that it achieves all the security properties: semantic security, anonymity, KwrtA-anonymity and identity-based non-malleability. For the sake of simplicity, we use a symmetric pairing:

Setup $_{\mathrm{IBK}}$. The setup algorithm chooses two random generators $g, h \in \mathbb{G}$, and a random exponent $\omega \in \mathbb{Z}_{p}$. It keeps this exponent as the master key $\mathrm{MK}=\omega$. The corresponding system parameters are: $\mathrm{PK}=$ $\left(g, g_{1}=g^{\omega}, h\right)$. It defines the identity-function: $F(\mathrm{ID})=g_{1} \cdot g^{\mathrm{ID}}=g^{\omega+\mathrm{ID}}$.

Note that, as above, the public parameters consist of independent elements in appropriate groups. The validity check Valid ${ }_{\mathrm{BBK}}(\mathrm{PK})$ is thus trivial.

Extract $_{\mathrm{IBK}}(\mathrm{MK}, \mathrm{ID})$. To issue a private key for identity ID, the key extraction authority computes the private key, usk $\mathrm{ID}_{\mathrm{D}}=h^{1 /(\omega+\mathrm{ID})}$.

Encaps $_{\mathrm{IBK}}(\mathrm{PK}, \mathrm{ID})$. In order to generate an ephemeral key with an identity ID, the algorithm chooses a random exponent $r \in \mathbb{Z}_{p}$, and creates the ciphertext as: $c=F(\mathrm{ID})^{r}$, that corresponds to the key $K=\hat{e}(g, h)^{r}$.

$\operatorname{Decaps}_{\mathrm{IBK}}\left(\right.$ usk $\left._{\mathrm{ID}}, c\right)$. The decryption algorithm extracts the ephemeral key $K$ from a ciphertext $c$ by computing: $K=\hat{e}\left(\right.$ usk $\left._{\mathrm{ID}}, c\right)$.

CorReCTNEss. Let us check the decryption process:

$$
K=\hat{e}\left(\text { usk }_{\mathrm{ID}}, c\right)=\hat{e}\left(h^{1 /(\omega+\mathrm{ID})}, g^{r(\omega+\mathrm{ID})}\right)=\hat{e}(h, g)^{r} .
$$

SEmantic SEcurity. It is worth to precise that we do not require to be able to simulate any oracle for making use of $\mathcal{I B}-\mathcal{K} \mathcal{E} \mathcal{M}$ schemes in the next section. The weak semantic security will be enough:

Theorem 3. The weak semantic security of our scheme (under selective-ID, chosen-plaintext and noidentity attacks) relies on the $\mathrm{DBDH}_{\mathbb{G}, \hat{e}}$-problem, in the standard model.

Proof. Given $u, A=u^{a}, B=u^{b}, C=u^{c}$, and $V \in \mathbb{G}_{T}$ the input to the $\mathrm{DBDH}_{\mathbb{G}, \hat{e}}-$ Problem, and the target identity ID*, we set $g=A=u^{a}, h=C=u^{c}=g^{c / a}, g_{1}=u^{t} \cdot A^{-\mathrm{ID}^{*}}=u^{t-a \mathrm{ID}^{*}}$, and $c=B$. This implicitly defines $\mathrm{MK}=t / a-\mathrm{ID}^{*}$, for a randomly chosen $t \stackrel{R}{\leftarrow} \mathbb{Z}_{p}$. Therefore, $F_{\mathrm{PK}}\left(\mathrm{ID}^{*}\right)=g_{1} g^{\mathrm{ID}^{*}}=u^{t} \cdot A^{-\mathrm{ID}^{*}} \cdot A^{\mathrm{ID}^{*}}=$ $u^{t}$, and the randomness $r$ of the challenge ciphertext $c=F_{\mathrm{PK}}\left(\mathrm{ID}^{*}\right)^{r}=u^{t r}=u^{b}=B$ is $r=b / t$. The corresponding encapsulated key should thus be

$$
K=\hat{e}(h, g)^{r}=\hat{e}\left(u^{c}, u^{a}\right)^{b / t}=\hat{e}(u, u)^{a b c / t} .
$$

By letting $\left(V^{1 / t}, c\right)$ be the output of the challenger, an adversary able to break the semantic security (without Extract-queries) helps us to decide whether $V$ is the Bilinear Diffie-Hellman value or not. 
In order to show the usual semantic security (under full-ID, but chosen-plaintext attacks), we have to be able to simulate the Extract-oracle, which thus requires additional inputs. But first, we modify a little bit the scheme, by using $H($ ID), instead of ID in the above description, where $H$ is a random oracle [5] onto $\mathbb{Z}_{p}$.

Theorem 4. The semantic security of our scheme (by using H(ID), instead of ID) under full-ID and chosen-plaintext (no Decaps queries) relies on the successive-power version, in the random oracle model.

Proof. Given $u, A=u^{a}, B=u^{b}, C=u^{c}, C_{i}=C^{1 / a^{i}}$, for $i=1, \ldots, q$, and $V \in \mathbb{G}_{T}$ the input to the $q-\mathrm{SP}-\mathrm{DBDH} \mathrm{G}_{\mathbb{G}, \hat{e}}$-problem, we first compute $\left\{V_{i}=\hat{e}(u, u)^{b c / a^{i}}\right\}_{i=0 \ldots q}$, since $V_{0}=\hat{e}(B, C)$, and $V_{i}=\hat{e}\left(B, C_{i}\right)$, for $i=1, \ldots, q$. Then, we set $g=A=u^{a}$ and $g_{1}=u^{t} \cdot A^{-x^{*}}$, for randomly chosen $t, x^{*} \stackrel{R}{\leftarrow} \mathbb{Z}_{p}$. This implicitly defines $\mathrm{MK}=t / a-x^{*}$. We also choose random elements $x_{1}, \ldots, x_{q} \stackrel{R}{\leftarrow} \mathbb{Z}_{p}^{*}$, and set $P(X)=$ $\prod\left(t X+x_{i}\right)$, a polynomial of degree $q$, where the number of random oracle queries is $q+1$. We then set $h=C^{P(1 / a)}=u^{c P(1 / a)}$, which can be easily computed granted $C, C_{1}, \ldots, C_{q}$.

First, all the random oracle queries will be answered by an $x^{*}+x_{i}$, or $x^{*}$ (for a unique randomly chosen query): we hope to assign $x^{*}$ to $H\left(\right.$ ID $\left.^{*}\right)$, the target identity, which happens with probability $1 / q$. Let us assume this situation:

- By definition, as above, $F_{\mathrm{PK}}\left(\mathrm{ID}^{*}\right)=g_{1} g^{H\left(\mathrm{ID}^{*}\right)}=u^{t} \cdot A^{-x^{*}} \cdot A^{x^{*}}=u^{t}$;

- For all the other identities, $H\left(\mathrm{ID}_{j}\right)=x_{j}$, and then usk $_{j}$ can be computed as

$$
h^{1 /\left(\mathrm{MK}+x^{*}+x_{j}\right)}=C^{P(1 / a) /\left(\mathrm{MK}+x^{*}+x_{j}\right)}=C^{P(1 / a) /\left(t / a+x_{j}\right)}=C^{P_{j}(1 / a)},
$$

where $P_{j}$ is a polynomial of degree $q-1$. Then usk ${ }_{j}$ can be easily computed granted $C, C_{1}, \ldots, C_{q-1}$. Hence the simulation of the Extract-oracle.

As above, the challenge ciphertext is set $c=B=u^{b}=F_{\mathrm{PK}}\left(\mathrm{ID}^{*}\right)^{r}$ for $r=b / t$. The corresponding encapsulated key should thus be

$$
K=\hat{e}(g, h)^{r}=\hat{e}\left(u^{a}, u^{c P(1 / a)}\right)^{b / t}=\left(\hat{e}(u, u)^{a b c}\right)^{P(1 / a) / t} .
$$

Let us expand $P(X)=\sum_{i=0}^{i=q} p_{i} X^{i}$, and then

$$
K=\hat{e}(u, u)^{a b c \cdot p_{0} / t} \times \prod_{i=1}^{i=q} \hat{e}(u, u)^{b c / a^{i-1} \cdot p_{i} / t}=\left(\hat{e}(u, u)^{a b c}\right)^{p_{0} / t} \times \prod_{i=1}^{i=q} V_{i-1}^{p_{i} / t} .
$$

If $V=\hat{e}(u, u)^{a b c}$, the correct key is $V^{p_{0} / t} \times \prod_{i=1}^{i=q} V_{i-1}^{p_{i} / t}$. In the random case, the same computation leads to a totally random key $\left(\right.$ note that $\left.p_{0}=\prod x_{i} \neq 0 \bmod p\right)$. Then, by letting $\left(V^{p_{0} / t} \times \prod_{i=1}^{i=q} V_{i-1}^{p_{i} / t}, c\right)$ be the output of the challenger, an adversary able to break the semantic security helps us to decide whether $V$ is the Bilinear Diffie-Hellman value or not. We thus break the $q$-SP-DBDH $\mathbb{G}_{\mathbb{G}, \hat{e}}$-problem.

Anonymity. The usual anonymity notion relies on the same assumption as the semantic security. Since the ciphertext consists of $c=F(\text { ID })^{r}$, a random element in $\mathbb{G}$, whatever the identity ID. It is thus clearly KwrtA-anonymous, in the information-theoretical sense.

Theorem 5. Our scheme is unconditionally KwrtA-anonymous. 


\begin{tabular}{|c|c|c|}
\hline \multicolumn{2}{|l|}{ Client $C$} & Server $S$ \\
\hline$p w \in \mathrm{Dic}$ & & $p w \in \mathrm{Dic}$ \\
\hline accept $\leftarrow$ false & & accept $\leftarrow$ false \\
\hline Valid(PK)? & $S, \mathrm{PK}$ & $(\mathrm{PK}, \mathrm{MK}) \leftarrow \operatorname{Setup}(\lambda)$ \\
\hline$(K, c) \leftarrow \operatorname{Encaps}(\mathrm{PK}, p w)$ & $C, c$ & $\begin{aligned} \text { usk } & \leftarrow \operatorname{Extract}(\mathrm{MK}, p w) \\
K^{\prime} & \leftarrow \operatorname{Decrypt}(\text { usk }, c)\end{aligned}$ \\
\hline AuthS $^{\prime}=\mathcal{H}_{1}(S, C, \mathrm{PK}, c, p w, K)$ & $S$, AuthS & AuthS $=\mathcal{H}_{1}\left(S, C, \mathrm{PK}, c, p w, K^{\prime}\right)$ \\
\hline $\begin{array}{r}\text { AuthS } \stackrel{?}{=} \text { AuthS' } \\
\text { If no error } / \text { reject } \\
\text { accept } \leftarrow \text { true } \\
\text { AuthC }=\mathcal{H}_{2}(S, C, \mathrm{PK}, c, p w, K)\end{array}$ & & \\
\hline $\mathrm{sk}=\mathcal{H}_{0}(S, C, \mathrm{PK}, c, p w, K)$ & $\underset{C, \text { AuthC }}{\longrightarrow}$ & $\begin{array}{c}\text { AuthC }{ }^{\prime}=\mathcal{H}_{2}\left(S, C, \mathrm{PK}, c, p w, K^{\prime}\right) \\
\text { AuthC } \stackrel{?}{=} \text { AuthC } \\
\text { If no error } / \text { reject } \\
\text { accept } \leftarrow \text { true } \\
\text { sk }=\mathcal{H}_{0}(S, C, \mathrm{PK}, c, p w, K)\end{array}$ \\
\hline
\end{tabular}

Fig. 1. IBK-PAKE: a Password-Authenticated Key-Exchange Protocol

IDENDITY-BASED NON-MALlEABILITy. Let us consider the ciphertext $c$, and its decryption with respect to $\mathrm{ID}_{i}$ for $i \in\{0,1\}$. In the following, $r_{i}$ is formally defined by $c=F\left(\mathrm{ID}_{i}\right)^{r_{i}}$, and $K_{i}=\hat{e}(g, h)^{r_{i}}$. Thus, the identity-based non-malleability relies on the intractability of finding $c,\left\{\mathrm{ID}_{i}, K_{i}\right\}$, with $\mathrm{ID}_{0} \neq \mathrm{ID}_{1}$ such that $r_{i}=\log _{\hat{e}(g, h)}\left(K_{i}\right)=\log _{F\left(\mathrm{ID}_{i}\right)}(c)$. This thus leads to a solution of the Common co-CDH-Problem.

Theorem 6. The identity-based non-malleability of our scheme relies on the Common co-CDH-Problem in groups $\mathbb{G}$ and $\mathbb{G}_{T}$.

\section{$4 \quad \mathcal{I} \mathcal{B K}-\mathcal{P} \mathcal{A K} \mathcal{E}:$ Our PAKE Protocol}

The previous sections focused on identity-based key encapsulation mechanisms, and new anonymity properties. We now show how a weakly semantically secure $\mathcal{I B}-\mathcal{K} \mathcal{E} \mathcal{M}$, that is both KwrtA-anonymous and identity-based non-malleable, can be used to build a password-authenticated key exchange.

\subsection{Description of our Scheme}

Our new scheme is generic. It basically consists in generating the session key using this $\mathcal{I} \mathcal{B}-\mathcal{K} \mathcal{E} \mathcal{M}$, under the common password as the identity, see Figure 1. The other party can easily recover the session key. Security notions for semantic security and perfect forward secrecy follow from the (weak) semantic security and anonymity properties of the $\mathcal{I B}-\mathcal{K} \mathcal{E} \mathcal{M}$ scheme.

\subsection{Security Analysis}

Communication Model. We assume to have a fixed set of protocol participants, and each of them can be either a client or a server. They are all allowed to participate to several different, possibly concurrent, 
executions of the key exchange protocol. We model this by allowing each participant an unlimited number of instances able to initiate or participate to executions of the protocol.

In the password-based scenario, the two parties share a low-entropy secret $p w$ which is drawn from a small dictionary Dic. In the following, we assume that the distribution is uniform. More complex distributions could be considered.

We use the security model introduced by Bellare et al. [4], improved by Abdalla et al. [2] to consider the Real-or-Random security notion instead of the Find-then-Guess. In this model, the adversary $\mathcal{A}$ has the entire control of the network, which is formalized by allowing $\mathcal{A}$ to ask the following query, $\operatorname{Send}(U, m)$, that models $\mathcal{A}$ sending the message $m$ to instance $U$. The adversary $\mathcal{A}$ gets back the response $U$ generates in processing the message $m$ according to the protocol. A query Send $(U$, INIT) initializes the key exchange algorithm, by activating the first player in the protocol.

From the original security model, we suppress the Execute-queries. Even if they were important to model passive attacks vs. active attacks, we consider a stronger security model where the adversary always uses Send-queries, either for simply forwarding a flow generated by a honest user, or for modifying/manufacturing a flow. Thereafter, if the whole transcript of an execution of the protocol turns out to consist of forwarded flows only, this execution is then considered as a passive attack: it is similar to an Execute-query in previous models [4]. If one flow has been modified or manufactured, the session corresponds to an active attack.

As a consequence, in addition to the usual security model with Execute-queries, the adversary can adaptively decide, during an execution of the protocol, whether the session will correspond to a passive attack, or to an active one, and not from the beginning of the session only (as in [18]). An attack game will consist of a mix of passive and active attacks, in a concurrent manner.

However, as usual, we will be essentially interested in active attacks: $q_{\text {activec }}$ and $q_{\text {actives }}$ will, respectively, denote the number of active attacks in which the adversary played against the client and the server, respectively. We want to show that $q_{\text {activec }}+q_{\text {actives }}$ is an upper-bound on the number of passwords the adversary may have tried.

Security Notions. Two main security notions have been defined for key exchange protocols. The first is the semantic security of the key, which means that the exchanged key is unknown to anybody other than the players. The second one is unilateral or mutual authentication, which means that either one, or both, of the participants actually know the key. In the following, we focus on the semantic security, also known as AKE Security.

The semantic security of the session key is modeled by an additional query Test $(U)$. Since we are working in the Real-or-Random scenario, this Test-query can be asked as many times as the adversary $\mathcal{A}$ wants, but to fresh instances only. The freshness notion captures the intuitive fact that a session key is not "obviously" known to the adversary. More formally an instance is said to be fresh if it has successfully completed execution and

1. Neither it nor its partner was corrupted before the session started

2. or, the attack, on this session, was passive.

Two instances are partners if they run a key exchange protocol together. This is formally modeled by the notion of session ID: the session ID is a string defined from the transcript (usually, it consists of the first flows, sent and received), and two instances are partners if they share the same session IDs.

The Test-query is answered as follows: a (private) coin $b$ has been flipped once for all at the beginning of the attack game, if $b=1$ (Real), then the actual session key sk is sent back, if $b=0$ (Random), or a 
random value is returned. Note that for consistency reasons, in the random case, the same random value is sent to partners.

We denote the AKE advantage as the probability that $\mathcal{A}$ correctly guesses the value of $b$ with its output $b^{\prime}: \operatorname{Adv}^{\text {ake }}(\mathcal{A})=2 \operatorname{Pr}\left[b=b^{\prime}\right]-1$.

The adversary will also have access to the Corrupt-query that leaks the password: it is useful to model the perfect forward secrecy. The latter notion means that a session key remains secret even after the leakage of the long-term secret.

Security Result. For our protocol, we can state the following security result, which proof can be found in the full version.

Theorem 7 (AKE Security). Let us consider an Identity-Based Key Encapsulation Mechanism IBK = (Setup, Extract, Encaps, Decaps) that is weakly semantically secure (selective-ID, chosen-plaintext attacks and no Extract-queries), anonymous, KwrtA-anonymous, and identity-based non-malleable, then our protocol IBK-PAKE, provides semantic security and perfect forward secrecy:

$$
A d v_{\text {ibk-pake }}^{\text {ake }}(\mathcal{A}) \leq 4 \times \frac{q_{\text {active }}}{N}+\operatorname{negl}(),
$$

where $q_{\text {active }}=q_{\text {activec }}+q_{\text {actives }}$ is the number of active attacks and $N$ is the size of the dictionary.

\section{Conclusion}

In this paper, we have first introduced two new security notions for identity-based key encapsulation mechanisms: the first one is an enhancement of the usual anonymity, the second one formalizes a kind on non-malleability, with respect to the recipient identity.

Then, we proposed the first scheme that is full-ID semantically secure against chosen-message attacks, and that achieves our new security notions.

We furthermore showed that these new security notions could be useful for identity-based schemes as a tool: we provided a new framework for password-authenticated key exchange, with an identity-based key encapsulation mechanism as a core sub-routine.

\section{Acknowledgment}

We would like to thank the anonymous referees for their fruitful comments. This work has been partially supported by European Commission through the IST Program under Contract IST-2002-507932 ECRYPT, and by the French ANR-07-SESU-008-01 PAMPA Project.

\section{References}

1. Michel Abdalla, Mihir Bellare, Dario Catalano, Eike Kiltz, Tadayoshi Kohno, Tanja Lange, John Malone-Lee, Gregory Neven, Pascal Paillier, and Haixia Shi. Searchable encryption revisited: Consistency properties, relation to anonymous IBE, and extensions. In CRYPTO 2005, LNCS 3621, pages 205-222. Springer, 2005.

2. Michel Abdalla, Pierre-Alain Fouque, and David Pointcheval. Password-based authenticated key exchange in the threeparty setting. In PKC 2005, LNCS 3386, pages 65-84. Springer, 2005.

3. Mihir Bellare, Alexandra Boldyreva, Anand Desai, and David Pointcheval. Key-privacy in public-key encryption. In ASIACRYPT 2001, LNCS 2248, pages 566-582. Springer, 2001.

4. Mihir Bellare, David Pointcheval, and Phillip Rogaway. Authenticated key exchange secure against dictionary attacks. In EUROCRYPT 2000, LNCS 1807, pages 139-155. Springer, 2000. 
5. Mihir Bellare and Phillip Rogaway. Random oracles are practical: A paradigm for designing efficient protocols. In $A C M$ CCS 93, pages 62-73. ACM Press, 1993.

6. Steven M. Bellovin and Michael Merritt. Encrypted key exchange: Password-based protocols secure against dictionary attacks. In 1992 IEEE Symposium on Security and Privacy, pages 72-84. IEEE Computer Society Press, 1992.

7. Kamel Bentaha, Pooya Farshim, John Malone-Lee, and Nigel P. Smart. Generic constructions of identity-based and certificateless KEMs. Journal of Cryptology, 21(2):178-199, April 2008.

8. Dan Boneh and Xavier Boyen. Efficient selective-ID secure identity based encryption without random oracles. In EUROCRYPT 2004, LNCS 3027, pages 223-238. Springer, 2004.

9. Dan Boneh and Xavier Boyen. Short signatures without random oracles. In EUROCRYPT 2004, LNCS 3027, pages 56-73. Springer, 2004.

10. Dan Boneh and Matthew K. Franklin. Identity-based encryption from the Weil pairing. In CRYPTO 2001, LNCS 2139, pages 213-229. Springer, 2001.

11. Dan Boneh, Ben Lynn, and Hovav Shacham. Short signatures from the Weil pairing. In ASIACRYPT 2001, LNCS 2248, pages 514-532. Springer, 2001.

12. Dan Boneh and Brent R. Waters. Conjunctive, subset, and range queries on encrypted data. Cryptology ePrint Archive, 2006.

13. Xavier Boyen and Brent Waters. Anonymous hierarchical identity-based encryption (without random oracles). In CRYPTO 2006, LNCS 4117, pages 290-307. Springer, 2006.

14. Dario Catalano, David Pointcheval, and Thomas Pornin. IPAKE: Isomorphisms for password-based authenticated key exchange. Journal of Cryptology, 20(1):115-149, January 2007.

15. Clifford Cocks. An identity based encryption scheme based on quadratic residues. In Cryptography and Coding, 8th IMA International Conference, LNCS 2260, pages 360-363. Springer, 2001.

16. Craig Gentry. Practical identity-based encryption without random oracles. In EUROCRYPT 2006, LNCS 4004, pages 445-464. Springer, 2006.

17. Shafi Goldwasser and Silvio Micali. Probabilistic encryption. Journal of Computer and System Sciences, 28(2):270-299, 1984.

18. David Pointcheval and Sébastien Zimmer. Multi-factor authenticated key exchange. In ACNS 2008, LNCS 5037, pages 277-295. Springer, 2008.

19. Adi Shamir. Identity-based cryptosystems and signature schemes. In CRYPTO '84, LNCS 196, pages 47-53. Springer, 1985.

20. Victor Shoup. Using hash functions as a hedge against chosen ciphertext attack. In EUROCRYPT 2000, LNCS 1807, pages 275-288. Springer, 2000.

21. Victor Shoup. ISO 18033-2: An emerging standard for public-key encryption. December 2004. Final Committee Draft.

\section{A Analysis in the Generic Model}

\section{A.1 The Common co-CDH-Problem}

Let us first recall the Common co-CDH-Problem: given $g, h \in \mathbb{G}$, and $V \in \mathbb{G}_{T}$, output $c \in \mathbb{G}, k_{0} \neq k_{1} \in \mathbb{Z}_{p}$, and $K_{0}, K_{1} \in \mathbb{G}_{T}$ such that:

$$
g h^{k_{i}}=\mathrm{co}^{-} \mathrm{CDH}_{K_{i}, c}(V) \text { for } i=0,1 .
$$

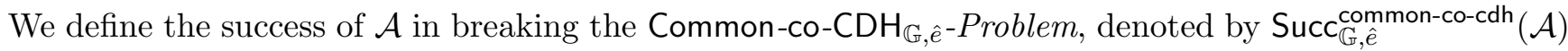
as:

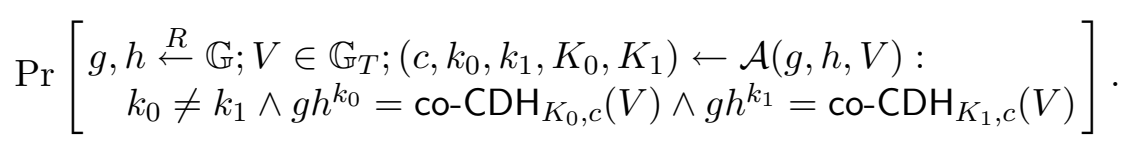

Theorem 8. Let $\mathcal{A}$ be an adversary that makes at most $q$ group operation queries (internal laws in $\mathbb{G}$ or $\mathbb{G}_{T}$, or pairing operations). On inputs $g, h \in \mathbb{G}$, and $V \in \mathbb{G}_{T}$, the probability that $\mathcal{A}$ outputs a solution $\left(k_{0}, k_{1}, K_{0}, K_{1}, c\right)$ to the Common co-CDH-Problem is bounded by

$$
\frac{(3 q+4)^{2}+3}{p} \leq \mathcal{O}\left(\frac{q^{2}}{p}\right) .
$$


Proof. Let $\mathcal{A}$ be an adversary against the Common co-CDH-Problem. We define a simulator $\mathcal{B}$ that emulates the group oracles: $\mathcal{B}$ maintains two lists $L_{1}$ and $L_{T}$ of polynomials $L_{1}=\left\{\left(F_{1, i}, \xi_{1, i}\right), i=1, \cdots, t_{1}\right\}$ and $L_{T}=\left\{\left(F_{T, i}, \xi_{T, i}\right), i=1, \cdots, t_{T}\right\}$ such that at step $t, t_{1}+t_{T} \leq 3 \cdot t+4$. The entries $\xi_{1, i}, \xi_{T, i}$ are set to be distinct random strings and are used to represent elements in $\mathbb{G}$ and $\mathbb{G}_{T}$ respectively. At the beginning of the game, $\mathcal{B}$ just sets two polynomials $F_{1,0}=1$ and $F_{1,1}=x_{1}$, which refer to a generator $g$ and a random element $h=g^{x_{1}}$ in $\mathbb{G}$, respectively. Similarly, $\mathcal{B}$ defines two polynomials $F_{T, 0}=1$ and $F_{T, 1}=X_{1}$ associated to elements $U=e(g, g)$ and $V=e(g, g)^{X_{1}}$ in $\mathbb{G}_{T}$.

For any oracle query, $\mathcal{B}$ updates the lists $L_{1}$ and $L_{T}$ :

- Group Operation in $\mathbb{G}$ : when $\mathcal{A}$ asks for the addition of two elements in $\mathbb{G}$, it gives two representations $\xi_{i}$ and $\xi_{j}$. Theses two strings are either associated to the polynomials $F_{1, i}, F_{1, j}\left(\left(F_{1, i}, \xi_{i}\right)\right.$ and $\left(F_{1, j}, \xi_{j}\right)$ are in $\left.L_{1}\right)$ or one defines a new variable $x_{1, i}$ and set $F_{1, i}=x_{1, i}$ associated to $\xi_{i}$ and thus adds $\left(F_{1, i}, \xi_{i}\right)$ to $L_{1}$ (or for index $j$ ). We thus assume that $\left(F_{1, i}, \xi_{i}\right)$ and $\left(F_{1, j}, \xi_{j}\right)$ are in $L_{1}$.

Then, it computes the sum of the polynomials, $F_{1, k}=F_{1, i}+F_{1, j}$. If the resulting polynomial $F_{1, k}$ already appears in the list for some index $l \leq t_{1}$, then it sets $\xi_{1, k} \leftarrow \xi_{1, l}$, else it chooses a new random string in $\{0,1\}^{\log _{2} p}$ for $\xi_{1, k}$. Note that group operations in $\mathbb{G}$ result in multivariate polynomials of degree at most one in variables $x_{1}, \cdots, x_{m}$, for some integer $m \leq t_{1}$.

- Pairing: when $\mathcal{A}$ requests a pairing query. It gives two representations $\xi_{1, i}$ and $\xi_{1, j}$. As above, by possibly setting the undefined elements, we can assume that $\left(F_{1, i}, \xi_{i}\right)$ and $\left(F_{1, j}, \xi_{j}\right)$ are in $L_{1}$. Then, $\mathcal{B}$ computes the product of the polynomials, $F_{T, k}=F_{1, i} \cdot F_{1, j}$. If the resulting polynomial already appears in the list for some index $l \leq t_{T}$, then it sets $\xi_{T, k} \leftarrow \xi_{T, l}$, else it chooses a new random string $\xi_{T, k}$ in $\{0,1\}^{\log _{2} p}$ for $F_{T, k}$.

Since we know that polynomials in $L_{1}$ are of degree 1 in the variables $x_{1}, \ldots$, the polynomials we create with this simulation are of degree 2 in the same variables.

- Group operation in $\mathbb{G}_{T}$ : when $\mathcal{A}$ asks for the addition of two elements in $\mathbb{G}_{T}$, it gives two representations $\xi_{i}$ and $\xi_{j}$. As above, by possibly setting the undefined elements (and new variables $X_{i}$ or $X_{j}$ ), we can assume that $\left(F_{T, i}, \xi_{i}\right)$ and $\left(F_{T, j}, \xi_{j}\right)$ are in $L_{T}$. Then, $\mathcal{B}$ computes the sum of the polynomials, $F_{T, k}=F_{T, i}+F_{T, j}$. If the resulting polynomial already appears in the list for some index $l \leq t_{T}$, then it sets $\xi_{T, k} \leftarrow \xi_{T, l}$, else it chooses a new random string $\xi_{T, k}$ in $\{0,1\}^{\log _{2} p}$ for $F_{T, k}$.

In the previous simulation, we created polynomials in $L_{T}$ of degree 2 in the variables $x_{1}, \ldots$ We can thus add these polynomials: they remain polynomials of degree 2 in the variables $x_{1}, \ldots$ We can also add these polynomials with the initial polynomials $F_{T, 1}, F_{T, 2}, \ldots$ and the new variables $X_{i}$ : polynomials of degree 1 in the variables $X_{1}, \ldots$

As a consequence, any polynomial $F$ in $L_{T}$ can be split in two polynomials $A \in \mathbb{Z}_{p}\left[x_{1}, \cdots, x_{m}\right]$ (of degree 2 ) and $B \in \mathbb{Z}_{p}\left[X_{1}, \cdots, X_{n}\right]$ (of degree 1) such that $F=A+B$.

Note that for each group operation query, the oracle adds at most three new variables in the list. Thus if $q$ is the number of queries we have $t_{1}+t_{T} \leq 3 q+4$.

To evaluate the success of any adversary in distinguishing the above simulation from the real oracles, one has to define the event raised in case of deviation. This happens if the evaluations of two polynomials on the initial vector $\left(x_{1}, \ldots, X_{1}, \ldots\right)$ refer to the same value: the oracles would output the same representation whereas our simulation just compares the polynomials and would thus output different representations. More precisely, the simulation can be detected if there exists a pair of polynomials $\left(F, F^{\prime}\right)$ such that for a random choice of $x_{1}, \cdots, x_{n}, X_{1}, \cdots, X_{m}$ in $\mathbb{Z}_{p}$,

$$
F\left(x_{1}, \cdots, x_{n}, X_{1}, \cdots, X_{m}\right)=F^{\prime}\left(x_{1}, \cdots, x_{n}, X_{1}, \cdots, X_{m}\right) \text { whereas } F \neq F^{\prime}
$$


Since polynomials are of degree at most 2 , this can happen with probability less than $2 / p$ for each pair of polynomials: after $q$ queries, the probability that the adversary distinguishes the two executions is bounded by $2 \cdot(3 q+4)^{2} / p$.

Unless the adversary $\mathcal{A}$ detects the simulation, it terminates by outputting a tuple $\left(k_{0}, k_{1}, \xi_{0}^{T}, \xi_{1}^{T}, \xi_{c}^{1}\right)$, where $k_{0}, k_{1}$ are in $\mathbb{Z}_{p} . \mathcal{B}$ retrieves, in the list, the polynomials associated to $\xi_{0}^{T}$ (representation of $K_{0}$ ), $\xi_{1}^{T}$ (representation of $K_{1}$ ) and $\xi_{c}^{1}$ (representation of $c$ ), if they exist. Otherwise, as before, it adds new variables. Let thus $F_{0}, F_{1}$ and $P$ be the polynomials associated to $\xi_{0}^{T}, \xi_{1}^{T}$ and $\xi_{c}^{1}$ respectively: $P \in \mathbb{Z}_{p}\left[x_{1}, x_{2}, \cdots, x_{m}\right]$ of degree one, and $F_{i} \in \mathbb{Z}_{p}\left[x_{1}, \cdots, x_{m}, X_{1}, \cdots, X_{n}\right]$ of degree two. More precisely, as noted before, we can split $F_{i}=A_{i}+B_{i}$, with $A_{i} \in \mathbb{Z}_{p}\left[x_{1}, x_{2}, \cdots, x_{m}\right]$ of degree two, and $B_{i} \in \mathbb{Z}_{p}\left[X_{1}, \cdots, X_{n}\right]$ of degree one.

If $\mathcal{A}$ is successful, this means that for some $\beta_{i}$, we have:

$$
c^{\beta_{i}}=g h^{k_{i}} \text { and } V^{\beta_{i}}=K_{i}
$$

The equalities above implies the following ones:

$$
\left\{\begin{array}{c}
\beta_{i} \cdot P\left(x_{1}, x_{2}, \cdots, x_{n}\right)=1+k_{i} x_{1} \\
X_{1} \cdot \beta_{i}=A_{i}\left(x_{1}, x_{2}, \cdots, x_{n}\right)+B_{i}\left(X_{1}, \cdots, X_{m}\right)
\end{array}\right.
$$

After substitution, we obtain

$$
\left(A_{i}\left(x_{1}, x_{2}, \cdots, x_{m}\right)+B_{i}\left(X_{1}, \cdots, X_{n}\right)\right) \cdot P\left(x_{1}, x_{2}, \cdots, x_{m}\right)-\left(1+k_{i} x_{1}\right) \cdot X_{1}=0
$$

At this point, either the success probability of the adversary is negligible (the above polynomial is non-zero), or

$$
A_{i}\left(x_{1}, x_{2}, \cdots, x_{m}\right)=0, \quad B_{i}\left(X_{1}, X_{2}, \cdots, X_{n}\right)=\beta_{i} \cdot X_{1}
$$

where $\beta_{i}$ is now known to be a constant. Since $P$ is a common polynomial, one gets

$$
\left(1+k_{1} x_{1}\right) \cdot \beta_{0}-\left(1+k_{0} x_{1}\right) \cdot \beta_{1}=0
$$

Again, either the success probability of the adversary is negligible (the above polynomial is non-zero), or $\beta_{0}=\beta_{1}$ and $k_{1} \beta_{0}=k_{0} \beta_{1}$, which implies that $k_{0}=k_{1}$. However, a successful attack does not allow that, which concludes the proof.

\section{B Analysis of the Successive-Power Problem.}

The Successive-Power problem is the following: given $g, g^{x}, g^{y}, g^{z}$, and $g^{z / x}, g^{z / x^{2}}, \ldots, g^{z / x^{q}}$, as well as $V$, from some $V \in \mathbb{G}_{T}$, where $q$ is a parameter, decide whether $V=\hat{e}(g, g)^{x y z}$, or $V$ is a random element of $\mathbb{G}_{T}$.

Theorem 9. Let $\mathcal{A}$ be an adversary that makes at most $t$ group operation queries. On input $g, g^{x}, g^{y}, g^{\frac{z}{x^{i}}}$, for $i \in\{0, \cdots, q\}$, the advantage of $\mathcal{A}$ in distinguishing the distribution of $V=\hat{e}(g, g)^{x y z}$ from the random distribution in $\mathbb{G}_{T}$ is bounded by

$$
\frac{(3 t+q+7)^{2}}{p} \leq \mathcal{O}\left(\frac{t^{2}+q^{2}}{p}\right)
$$


Proof. As in previous proof, we construct an algorithm $\mathcal{B}$ that interacts with $\mathcal{A}$, using lists of pairs $L_{1}=\left\{\left(F_{1, i}, \xi_{1, i}\right)\right\}$ and $L_{T}=\left\{\left(F_{T, i}, \xi_{T, i}\right)\right\}$, but this time, we use fractions of polynomials. It starts with $F_{1,1}=1, F_{1,2}=X, F_{1,3}=Y, F_{1, i}=\frac{Z}{X^{i-4}}$ for $i=\{4, \cdots, q+4\}$, and $F_{T, 1}=1, F_{T, 2}=T_{0}, F_{T, 3}=T_{1}$. $X, Y, Z$ are unknown variables. For a random bit $b, T_{b}$ is also a really new unknown variable, whereas $T_{1-b}=X Y Z$ (but considered as an independent variable too. The adversary has to guess $b$.

When $\mathcal{A}$ terminates, it outputs its guess $b^{\prime}$, and then $\mathcal{B}$ chooses a random assignment $x, y, z, t_{b} \in \mathbb{Z}_{p}$, for $X, Y, Z$, and $T_{b}$ but sets $T_{1-b}=x y z$.

In the simulated game, the advantage of the adversary is clearly zero: all the polynomials built during the simulation are independent to $X Y Z$.

One thus have just to evaluate the probability the adversary can detect that it is interacting with a simulator: after $t$ queries, the number of polynomials is upper-bounded by $3 t+q+7$, which concludes the proof. 